\title{
Sticky Platelet Syndrome
}

\author{
EBERHARD F. MAMMEN, M.D.
}

\begin{abstract}
The sticky platelet syndrome (SPS) is an autosomal dominant platelet disorder associated with arterial and venous thromboembolic events. It is characterized by hyperaggregability of platelets in platelet-rich plasma with adenosine diphosphate (ADP) and epinephrine (type I), epinephrine alone (type II), or ADP alone (type III). Clinically, patients may present with angina pectoris, acute myocardial infarction (MI), transient cerebral ischemic attacks, stroke, retinal thrombosis, peripheral arterial thrombosis, and venous thrombosis, frequently recurrent under oral anticoagulant therapy. Clinical symptoms, especially arterial, often present following emotional stress. Combinations of SPS with other congenital thrombophilic defects have been described. Low-dose aspirin treatment $(80$ to $100 \mathrm{mg})$ ameliorates the clinical symptoms and normalizes hyperaggregability. The precise etiology of this defect is at present not known, but receptors on the platelet surface may be involved. Normal levels of platelet factor 4 (PF4) and $\beta$-thromboglobulin in plasma suggest that the platelets are not activated at all times; they appear to become hyperactive upon ADP or adrenaline release. In vivo clumping could temporarily or permanently occlude a vessel, leading to the described clinical manifestations. The syndrome appears to be prominent especially in patients with unexplained arterial vascular occlusions.
\end{abstract}

Keywords: Platelets, platelet aggregation, ADP, epinephrine, thrombophilia, thrombosis

Thromboembolic disorders are the most common cause of death in the western world, and several hundred thousand persons die annually in the United States as a consequence of arterial or venous thromboembolism. ${ }^{1}$ Over the years a number of coagulation factor abnormalities have been identified that have been reviewed before ${ }^{3}$ and some were extensively discussed in the previous issue of Seminars in Thrombosis and Hemostasis (Volume 25, Number 3, 1999) and in this issue. Most of these de- fects relate to the inhibitor system of the clotting cascade, in other words, antithrombin, protein $\mathrm{C}$, protein $\mathrm{S}$, activated protein $\mathrm{C}$ (APC) resistance, and possibly thrombomodulin and heparin cofactor II, or the fibrinolytic system, in other words plasminogen activator inhibitor type I (PAI-1), tissue plasminogen activator (t-PA), and possibly urokinase plasminogen activator (u-PA). Additional defects relate to a gene mutation in the prothrombin molecule, in hyperhomocysteinemia, and in the presence of antiphos-

Objectives

Upon completion of the article the reader should be able to: 1) list the three forms of the sticky platelet syndrome, 2) consider the syndrome when patients present with predominantly arterial forms of thrombosis without identifiable risk factors, and 3) be able to initiate appropriate treatment modalities.

Accreditation

The Indiana University School of Medicine is accredited by the Accreditation Council for Continuing Medical Education to sponsor continuing medical education for physicians.

Credit

The Indiana University School of Medicine designates this educational activity for a maximum of 1.0 hours in category one credit toward the AMA Physicians Recognition Award.

Disclosure

Statements have been obtained regarding the author's relationships with financial supporters of this activity. There is no apparent conflict of interest related to the context of participation of the authors of this article. 
pholipid antibodies. All affect the clotting or fibrinolytic system, and surprisingly little is known about platelet defects that predispose to thromboembolic disorders, although platelets play a very important role in the pathogenesis of thrombosis, especially arterial events. ${ }^{4}$ This may, in part, be due to the difficult assays for platelet function; the techniques for assessing platelet adhesion and particularly platelet aggregation are labor intensive and thus expensive and are difficult to standardize. Platelet aggregometry was moreover primarily designed to measure decreased platelet function and not potentially increased responses. Until recently there was not even an accurate and reliable screening test for platelet function, and the most widely used bleeding time is of very limited usefulness. ${ }^{5}$ The more recently introduced PFA-100 system (platelet function analyzer) is far more accurate in diagnosing defects of primary hemostasis ${ }^{6}$ but has not yet been fully evaluated in its capacity to measure "hyperactive" platelets.

There have been numerous reports in the literature in which hyperaggregable platelets were found in a variety of diseases, such as unstable angina, diabetes mellitus, nephrotic syndrome, cystic fibrosis, migraine headaches, strokes, mitral valve prolapse, retinal artery thrombosis, anorexia nervosa, and acute thromboembolic events, ${ }^{7}$ but these seem to be acquired rather than congenital. In patients with these disorders and hyperaggregable platelets, plasma levels of PF4, $\beta$-thromboglobulin, and thromboxane $A_{2}$ were also elevated, which suggests that there is in vivo activation of platelets. Hyperaggregable platelets during catecholamine release but no clinical symptoms have also been reported ${ }^{8}$ and may be related to the pathogenesis of acute MI coinciding with the well-recognized circadian variation. ${ }^{9}$ Again, this is associated with in vivo platelet activation as judged by elevated plasma levels of PF4 and $\beta$-thromboglobulin.10

Congenital defects in the prostaglandin pathway associated with thrombosis, especially arterial, have been reported ${ }^{11}$ with no elevated plasma levels of PF4 and $\beta$ thromboglobulin. All of these patients were relatively young and had no other identifiable risk factors.

Again the reason for the paucity in platelet defects associated with thrombosis may to some extent reside in the technical difficulties associated with diagnosing such problems; there is no reason why they should not exist.

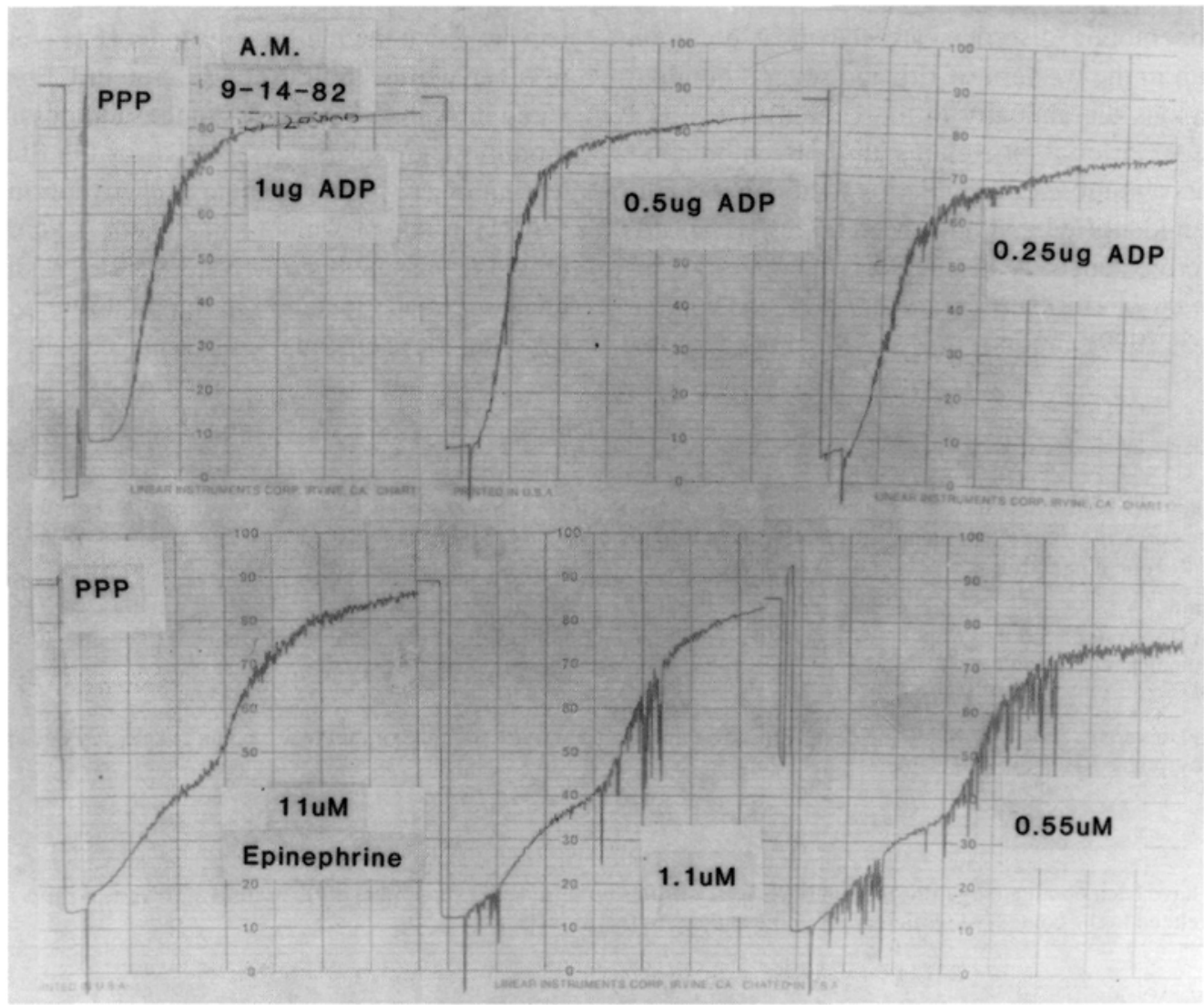

FIG. 1. Typical platelet aggregation patterns with ADP and epinephrine in a patient with SPS, type I. (Reproduced, with permission, from Mammen. ) 


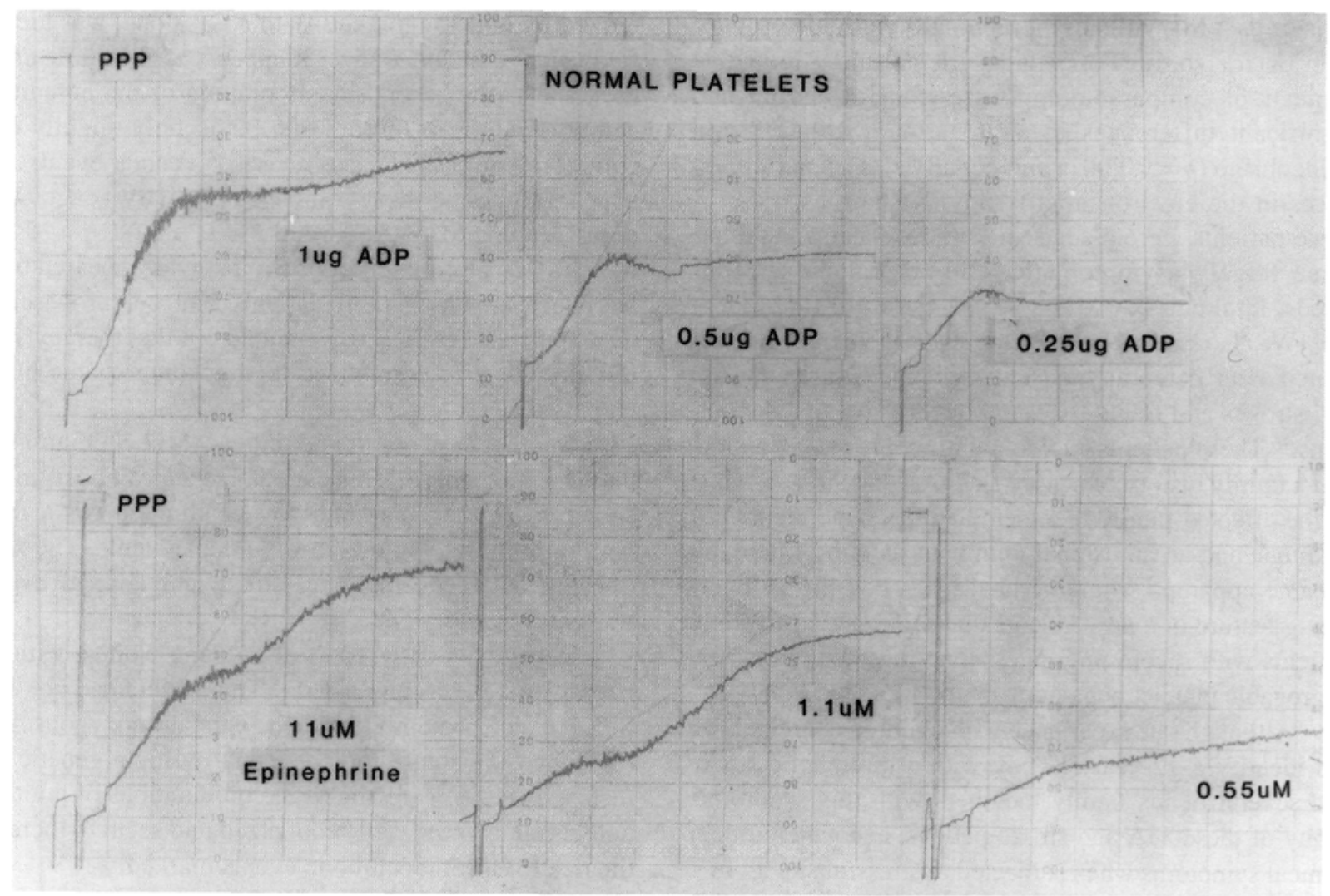

FIG. 2. Platelet aggregation patterns in a healthy person with dilutions of ADP and epinephrine. Note the dose-response pattern. (Reproduced, with permission, from Mammen. ${ }^{7}$ )

\section{DISCOVERY}

In 1982 a 24-year-old woman was referred to our laboratory for a hypercoagulability work-up. She had suffered an acute MI while 7 months pregnant. There were no identifiable risk factors or diseases, and coronary arteriography had revealed no evidence for atherosclerotic lesions. The family history indicated that the patient's mother had suffered an acute MI with one of her three pregnancies and that at that time an 18-year-old brother had angina pectoris attacks without identifiable coronary artery disease. The patient's father and one sister were clinically asymptomatic.

Basic coagulation tests and platelet counts were normal, and the known defects associated with familial thrombophilia (antithrombin, protein C, and protein S) were also normal. Routine platelet aggregation studies on platelet-rich plasma (PRP) using the usual concentrations of ADP, collagen, epinephrine, arachidonic acid, ristocetin, and thrombin were normal. Because the aggregation response to the standard concentrations of ADP and epinephrine (2.34 and $11 \mu \mathrm{M}$, respectively) were virtually $100 \%$, we decided to dilute both agents and made the observation shown in Figure 1. It can be seen that the dose-response observed with platelets from a healthy person (Fig. 2) was missing. The findings suggested that the patient's platelets were hyperaggregable with $\mathrm{ADP}$ and epinephrine. With the other agonists, no such difference was seen. The technical details of this testing have been extensively described elsewhere in the literature. 7,12

When we tested the patient's parents and siblings with the same approach, we found that the mother and the brother with angina attacks had the same aggregation responses, whereas the father and sister did not. This suggested the potential for a congenital defect.

These studies were repeated several times using different aggregometers and different techniques, and the data were always reproducible.

While studying platelet adhesiveness and aggregability in response to surface ${ }^{13}$ with electron microscopy, it was observed that the patient's platelets were both hyperadhesive and hyperaggregable. Measurements of PF4 and $\beta$-thromboglobulin in the patient's plasma were repeatedly negative.

The patient and her positive family members were put on aspirin ( $81 \mathrm{mg}$ every day), after which the hyperaggregable platelets became normal. Discontinuing aspirin once again revealed the hyperaggregable patterns.

\section{ADDITIONAL STUDIES}

The findings on our first patient prompted the cardiologist to refer additional patients with angina pec- 
toris or past MI with angiographically normal coronary arteries for study. Forty-one patients and 71 control subjects of comparable age were studied, and all had significant differences in aggregation with ADP and epinephrine $(p=<0.001)$ and equally significant differences in the electron microscopy evaluations. ${ }^{14}$ All of these patients were placed on low-dose aspirin and became largely asymptomatic. Only 1 patient encountered a fatal massive MI.

We also studied young patients ( $<45$ years) who presented with cerebral transient ischemic attacks (TIAs) and strokes and found the same hyperactive platelet patterns. ${ }^{15}$ These patients had no identifiable risk factors but had a family history of acute MI, TIAs, and strokes. Most of the affected family members had the same laboratory findings, and an autosomal dominant pattern of heredity became apparent. Migraine headaches became an interesting feature in many patients that prompted us to study patients with severe migraine. None revealed the hyperaggregable platelet behavior.

Although our experience with children is limited, we did identify a 12-year-old boy with a thrombotic stroke and several of his family members with this syndrome. Many of these TIA and stroke patients encountered their clinical symptoms when subjected to stressful situations.

In cooperation with an ophthalmologist, we studied 20 patients with "idiopathic," ischemic optic neuropathy who had temporary or permanent vision loss, usually in one eye. Twenty-seven age-, sex-, and race-matched controls were also tested. Again, significant $(p=<0.0001$, $0.001,0.01$, respectively) differences in ADP and epinephrine-induced aggregability were observed.7,12

During these studies, involving well over 200 patients by 1996, we identified two types of patients, one with the classic hyperaggregable platelets with ADP and epinephrine, now referred to as type I, and the other with hyperaggregability with epinephrine alone, called type II. The patients are clinically indistinguishable, and families have either type I or type II; we have not seen any mixed families.

We have also identified this syndrome in patients with venous thrombosis who experienced recurrence while on oral anticoagulants. Overall, in our experience, most patients had arterial forms of thrombosis; venous thromboses were rare by comparison.

\section{LITERATURE REPORTS}

In 1998, Bick published his experience with SPS. ${ }^{1}$ He began testing for it in 1995 and in 2 years studied 153 patients with unexplained arterial and venous thrombotic events. Twenty-one percent of patients with unexplained arterial events (acute MI, TIAs, thrombotic stroke, retinal thrombosis, and peripheral arterial thrombosis) had SPS, and $13.2 \%$ of patients had unexplained venous thrombosis. In this study, a type III SPS, charac- terized by hyperaggregability to ADP alone, was identified. Bick ${ }^{1}$ concluded that "it appears SPS is quite common and is the second most common of the hereditary defects leading to thrombosis, being only slightly less common than APC-R as a cause of venous events and appears to be the most common hereditary coagulation defect leading to arterial events."

In 1997, a case report of two patients appeared from Germany in which both patients had cerebrovascular thromboses. ${ }^{16}$ Again it was pointed out that therapy with low-dose aspirin normalized the hyperaggregable platelet pattern.

Another case report and review of the literature was published recently. ${ }^{17}$ This patient had a thrombosis in the superior sagittal sinus and was readmitted for a deep vein thrombosis while on oral anticoagulants. The relationship between emotional stress and clinical events was emphasized.

Recently, a case report of a young woman with bilateral strokes was published. ${ }^{18}$ This patient had not only SPS but also protein S deficiency and homozygous APC resistance. This case indicates that multiple genetic defects may accompany the SPS; combinations of multiple defects are by now well-recognized and seem to increase the risk for thromboembolic events markedly.

In December 1998, Andersen ${ }^{19}$ reported at a symposium held in conjunction with the 40th Annual Meeting of the American Society of Hematology her experience with 195 patients with arterial, venous, or mixed thromboses who were prospectively screened. Fifty-six (28\%) of the patients had SPS inherited as an autosomal dominant trait, $16 \%$ had hyperhomocysteinemia, $16 \%$ had PAI-1 excess, and 15\% had APC resistance. In 18 patients, the SPS coexisted with other congenital defects. Again, the SPS appears to be a prominent finding in patients with thromboembolic events.

\section{REFERENCES}

1. Bick RL. Sticky platelet syndrome: A common cause of unexplained arterial and venous thrombosis. Clin Appl Thromb Hemost 1998;4:77-81

2. Comp PC. Congenital and acquired hypercoagulable states. In: Hull R, Pineo GF, eds. Disorders of Thrombosis. Philadelphia, PA: Saunders; 1996;339-347

3. Middledorp S, Briët E, Conard J. Familial thrombophilia. In: Verstraete M, Fuster V, Topol EJ, eds. Cardiovascular Thrombosis. Thrombocardiology and Thromboneurology, 2nd ed. Philadelphia, PA: Lippincott-Raven; 1998;59-75

4. Badimon L, Badimon JJ, Fuster V. Pathogenesis of thrombosis. In: Verstraete M, Fuster V, Topal EJ, eds. Cardiovascular Thrombosis. Thrombocardiology and Thromboneurology, 2nd ed. Philadelphia, PA: Lippincott-Raven; 1998;23-44

5. Rodgers RPC, Levine J. A critical reappraisal of the bleeding time. Semin Thromb Hemost 1990;16:1-20

6. Mammen EF, Comp RC, Gosselin R, et al. PFA-100 ${ }^{\mathrm{TM}}$ system: A new method for assessment of platelet dysfunction. Semin Thromb Hemost 1998;24:195-202 
7. Mammen EF. Ten year's experience with the "sticky platelet syndrome." Clin Appl Thromb Hemost 1995;1:66-72

8. Roswell HC, Hegardt B, Downie HG, et al. Adrenalin and experimental thrombosis. Br J Haematol 1966; 12:66-71

9. Tofler GH, Brezinski D, Schafer AI, et al. Morning increase in platelet response to ADP and epinephrine: Association with the time of increased risk of myocardial infarction and sudden cardiac death. N Engl J Med 1987;316:1514-1519

10. Jafri SM, van Rollins M, Ozawa T, et al. Circadian variations in platelet function in healthy volunteers. Am J Cardiol 1992; 69:951-954

11. Sinzinger H, Kaliman J, O'Grady J. Platelet lypoxygenase defect (Wien-Penzing defect) in two patients with myocardial infarction. Am J Hematol 1991;36:202-205

12. Mammen EF, Barnhart MI, Selik NR, et al. "Sticky platelet syndrome": A congenital platelet abnormality predisposing to thrombosis? Folia Haematol (Leipzig) 1988;115:361-365

13. Barnhart MI, Gilroy J, Meyer JS. Dextran 40 in cerebrovascular thrombosis. Thrombosis et Diathesis Haemorrhagica 1970; 42(suppl):321-332
14. Rubenfire M, Blevins RD, Barnhart MI, et al. Platelet hyperaggregability in patients with chest pain and angiographically normal coronary arteries. Am J Cardiol 1986;57:657-660

15. Holliday PL, Mammen E, Gilroy J, et al. Sticky platelet syndrome and cerebral infarction in young adults. Presented at the Ninth International Joint Conference on Stroke and Cerebral Circulation; 1983; Phoenix, AZ

16. Berg-Damer E, Henkes H, Trobisch H, Kühne D. Sticky platelet syndrome: A cause of neurovascular thrombosis and thromboembolism. Intervent Neuroradiol 1997;3:145-154

17. Chittoor SR, Elsehety AE, Roberts GF, Laughlin WR. Sticky platelet syndrome: A case report and review of the literature. Clin Appl Thromb Hemost 1998;4:280-284

18. Chaturvedi S, Dzieczkowski JS. Protein S deficiency, activated protein $\mathrm{C}$ resistance and sticky platelet syndrome in a young woman with bilateral strokes. Cerebrovasc Dis 1999;9:127130

19. Andersen JA. Report. Bleeding and thrombosis in women. Biomed Progress 1999;12:40 